\title{
AN EXISTENCE THEOREM FOR THE REDUCED WAVE EQUATION
}

\author{
PETER WOLFE ${ }^{1}$
}

We consider here the two dimensional problem of scattering of a wave by a finite set of smooth, finite, nonintersecting arcs. Let the points in $E^{2}$ be denoted by $z=x+i y$. Let $L_{i}, i=1, \cdots, n$ be $\operatorname{arcs}$ given by

$$
z=x_{i}(t)+i y_{i}(t), \quad 0 \leqq t \leqq 1, \quad i=1, \cdots, n .
$$

We denote the point $x_{i}(0)+i y_{i}(0)$ by $a_{i}$ and the point $x_{i}(1)+i y_{i}(1)$ by $b_{i}$. We assume that the functions $x_{i}(t)$ and $y_{i}(t)$ have Hölder continuous second derivatives and that the $\operatorname{arcs} L_{i}$ do not intersect. We denote the union of the $L_{i}$ 's by $L$ and the open set $E^{2}-L$ by $G$. We seek a function $u_{s}(x, y)$ which satisfies the following conditions:

(a) $u_{s}$ is continuous in $E^{2}$,

(b) in $G, u_{s}$ satisfies the reduced wave equation

$$
\frac{\partial^{2} u_{s}}{\partial x^{2}}+\frac{\partial^{2} u_{s}}{\partial y^{2}}+k^{2} u_{s}=0, \quad k \neq 0, \quad \operatorname{Re} k \geqq 0, \quad \operatorname{Im} k \geqq 0,
$$

(c) $u_{s}$ satisfies the radiation condition

$$
\lim _{\rho \rightarrow \infty} \int_{|z|=\rho}\left|\frac{\partial u_{s}}{\partial|z|}-i k u_{s}\right|^{2} d s=0,
$$

(d) on $L, u_{s}+u_{0}=0$

where $u_{0}$ is a prescribed function (the incident wave) which satisfies (1) in all of $E^{2}$.

The purpose of this note is to prove the following

THEOREM. There exists a (unique) function $u_{s}$ satisfying conditions (a)-(d) above.

Uniqueness follows from the work of Levine [2]. (Levine proves his uniqueness theorem in the three dimensional case, however his proof can easily be modified so as to apply here.) Here we will prove existence. Our method is similar to that of Leis [1] who considered the case of scattering by a piecewise smooth closed contour.

Received by the editors August 12, 1968.

1 The research in this paper was supported by a Faculty Research Award by the General Research Board of the Graduate School, University of Maryland. 
Proof of the Existence Theorem. Let $z_{0}=x_{0}+i y_{0}$ and $r$ $=\left|z-z_{0}\right|$. Define

$$
Q\left(z, z_{0}\right)=(i / 4) H_{0}^{(1)}(k r),
$$

where $H_{0}^{(1)}$ denotes the zero order Hankel function of the first kind. Then for fixed $z_{0}$ and $z \neq z_{0} Q$ satisfies (1) and (2). For small $r$ we have

$$
(i / 4) H_{0}^{(1)}(k r)=(1 / 2 \pi) \log (1 / r)+h(r),
$$

where $h(r)$ and $h^{\prime}(r)$ are finite at $r=0$ (i.e. $Q\left(z, z_{0}\right)$ is the fundamental outgoing solution of the reduced wave equation).

We seek a solution to the problem of the form

$$
u_{s}(z)=\int_{L} \phi\left(z_{0}\right) Q\left(z, z_{0}\right) d s_{0} .
$$

We assume that the unknown function $\phi$ defined on $L$ is Hölder continuous in the neighborhood of every interior point of the $\operatorname{arcs} L_{i}$ and if $c_{i}$ is one of the endpoints of $L_{i}\left(c_{i}=a_{i}\right.$ or $\left.b_{i}\right)$ we have

$$
\left|\phi\left(z_{0}\right)\right| \leqq K /\left|z_{0}-c_{i}\right|^{\alpha}, \quad z_{0} \in L_{i} \quad 0 \leqq \alpha<1 .
$$

Functions satisfying the above conditions will be said to belong to class $h$. (We are motivated to seek $\phi$ out of this class of functions by examining the solution of the problem of diffraction by a half line.) Then the function $u_{s}$ defined by (5) will satisfy conditions (a), (b), and (c) of the problem. To satisfy the boundary condition (d) we must have

$$
-u_{0}(z)=\int_{L} \phi\left(z_{0}\right) Q\left(z, z_{0}\right) d s_{0}, \quad z \in L .
$$

By (4) $Q\left(z, z_{0}\right)$ has a logarithmic singularity at $z=z_{0}$. However, by a theorem in $[3$, p. 31] we may differentiate the right-hand side of (6) by differentiating formally under the integral sign and interpreting the result as a Cauchy Principal Value. Now

$$
\frac{\partial Q}{\partial s}=-\frac{1}{2 \pi} \frac{d \log r}{d s}+h^{\prime}(r) \frac{d r}{d s} .
$$

$r=\left(z-z_{0}\right) e^{-i \nu}$ where $\nu$ is the argument of the difference $z-z_{0}$. Hence

$$
\frac{d \log r}{d s}=\frac{1}{z-z_{0}} \frac{d z}{d s}-i \frac{d \nu}{d s}=\frac{1}{z-z_{0}} e^{i \theta(z)}-i \frac{d \nu}{d s}
$$

where $\theta(z)$ is the angle between the $x$ axis and the tangent to $L$ at $z$. 
Further $d s_{0}=\exp \left(-i \theta\left(z_{0}\right)\right) d z_{0}$. Thus the result can be written in the form

$$
f(z) \equiv-\frac{\partial u_{0}}{\partial s}(z)=\int_{L} \phi\left(z_{0}\right) \frac{K\left(z, z_{0}\right)}{z_{0}-z} d z_{0}
$$

with

$$
K\left(z, z_{0}\right)=\left\{\frac{1}{2 \pi} e^{i \theta(z)}+\frac{i}{2 \pi} \frac{d \nu}{d s}\left(z-z_{0}\right)+h^{\prime}(r) \frac{d r}{d s}\left(z_{0}-z\right)\right\} e^{-i \theta\left(z_{0}\right)} .
$$

$K\left(z, z_{0}\right)$ is Hölder continuous with respect to both variables on each of the closed arcs $L_{i}$ and $K\left(z_{0}, z_{0}\right) \neq 0, z_{0} \in L$. It is required then to find a solution $\phi$ in the class $h$ of the singular integral equation (7). $\phi$ is to be a solution in the sense that (7) holds for $z$ on $L$ except perhaps at end points.

Singular integral equations of the type (7) are treated in Chapter 14 of $[3] .^{2}$ If we write (7) as

$$
K \phi=f,
$$

we may state the results of [3] as applied to the present situation as follows.

1. The necessary and sufficient conditions of solubility in class $h$ of the equation (8) are $\int_{L} f(z) \Psi_{j}(z) d z=0$ where $\Psi_{j}\left(j=1, \cdots, k^{\prime}\right)$ is a complete system of linearly independent solutions in a certain class $h^{\prime}$ of an adjoint equation $K^{\prime} \psi=0$. (The definitions of $K^{\prime}$ and $h^{\prime}$ need not concern us here.)

2. If $k$ is the number of linearly independent solutions of the class $h$ of the homogeneous equation $K \phi=0$ and $k^{\prime}$ is the number of linearly independent solutions of the class $h^{\prime}$ of the adjoint equation $K^{\prime} \psi=0$, then $k-k^{\prime}=n$.

In the present case we shall show that $k=n$. Hence $k^{\prime}=0$ and thus (8) will have a solution for every (Hölder continuous) $f$.

Let $\phi_{j}$ be a solution of class $h$ of $K \phi=0$. Then we know that the function

$$
v_{j}(z)=\int_{L} \phi_{j}\left(z_{0}\right) Q\left(z, z_{0}\right) d s_{0}
$$

is constant on each $L_{i}$ since its derivative with respect to $s$ vanishes on each $L_{i}$. Define $c_{i j}$ by $v_{j}(z)=c_{i j}, z \in L_{i}$.

${ }^{2}$ In [3] the author considers a more general equation namely $A(z) \phi(z)+(1 / \pi i)$ - $\int_{L}\left(K\left(z, z_{0}\right) \phi\left(z_{0}\right) /\left(z_{0}-z\right)\right) d z_{0}=f(z)$. The basic assumption under which this equation is studied is that if $B(z)=K(z, z)$ then $A^{2}(z)-B^{2}(z) \neq 0$ for $z$ on $L$. In the present case we have $A(z)=0$. The above condition then obtains since we have $K(z, z)=0$. 
Now let $\phi_{1}, \cdots, \phi_{k}$ be a complete set of linearly independent solutions of $K \phi=0$. We have by statement 2 above $k \geqq n$. We now prove

Lemma 1. $k=n$ (hence $k^{\prime}=0$ ). Furthermore the rank of the matrix $\left(c_{i j}\right)$ is $n$.

Suppose the lemma were false. Then we could find constants $a_{j}, j$ $=1, \cdots, k$ not all zero such that

$$
\sum_{i=1}^{k} c_{i j} a_{j}=0, \quad i=1, \cdots, n .
$$

Consider the functions $\phi_{0}=\sum_{j=1}^{k} a_{j} \phi_{j}$ and $v_{0}=\int_{L} \phi_{0}\left(z_{0}\right) Q\left(z, z_{0}\right) d s_{0}$. On $L_{i}$ we have

$$
v_{0}(z)=\sum_{j=1}^{k} a_{j} \int_{L} \phi_{j}\left(z_{0}\right) Q\left(z, z_{0}\right) d s_{0}=\sum_{j=1}^{k} a_{j} c_{i j}=0 .
$$

Hence $v_{0}$ satisfies conditions (a), (b), (c) and vanishes on $L$. Thus by uniqueness $v_{0} \equiv 0$. But then $\phi\left(z_{0}\right)=[\partial v / \partial n]\left(z_{0}\right)=0$ where $\left[\partial v_{0} / \partial n\right]\left(z_{0}\right)$ denotes the jump in the normal derivative of $v_{0}$ across $L$ at the point $z_{0}$. Thus $\sum_{j=1}^{k} a_{j} \phi_{j} \equiv 0$, contradicting the linear independence of the $\phi_{j}$ 's. Hence we can solve (7) for any $f$. Let $\eta_{0}$ be a solution of (7) in the class $h$. Then we have

$$
u(z)=\int_{L} \eta_{0}\left(z_{0}\right) Q\left(z, z_{0}\right) d s_{0}=-u_{0}(z)+C_{i}, \quad z \in L_{i},
$$

where $C_{i}$ are definite constants. But by Lemma 1 we can determine constants $a_{1}, \cdots, a_{n}$ so that

$$
\sum_{j=1}^{n} c_{i j} a_{j}=-C_{i}, \quad i=1, \cdots, n .
$$

Then if $\eta=\eta_{0}+\sum_{j=1}^{n} a_{j} \phi_{j}, u_{s}(z)=\int_{L} \eta\left(z_{0}\right) Q\left(z, z_{0}\right) d s_{0}$ satisfies (6) and hence satisfies conditions (a) $-(d)$ of the problem.

\section{REFERENCES}

1. R. Leis, Über die Randwertaufgaben des Aussenraumes zur Helmholtzschen Schwingungsgleichung, Arch. Rational Mech. Anal. 9 (1962), 21-44.

2. L. Levine, $A$ uniqueness theorem for the reduced wave equation, Comm. Pure Appl. Math. 17 (1964), 147-176.

3. N. Muskhelishvili, Singular integral equations, Noordhoff, Groningen, 1953.

UNIVERSITY OF MARYLAND 